\title{
Evaluation of cancer rehabilitation in Austria
}

\author{
Richard Crevenna
}

Published online: 9 November 2017

(c) Springer-Verlag GmbH Austria 2017

Today, in Austria there are more than 600 beds for inpatient cancer rehabilitation which has become an important issue in the management of cancer patients [1, 2]. Cancer and its treatment can lead to pain, fatigue, nutritional deficits, and loss of muscle mass and decreased muscular strength and endurance capacity, impaired flexibility, decreased mobility, polyneuropathy, hand-foot syndrome, mucositis, lymphedema, incontinence, sexual dysfunction, cognitive deficits, dysthymia, and depression. As survival rates and survival time of cancer patients are increasing, cancer rehabilitation-using a rehabilitation process to counteract these sequelae and side effects-is an important part in the treatment and care of cancer patients with the goal to improve functional status (physical, mental, and psychosocial functions), quality of life, and participation $[1,2]$. Therefore, rehabilitation centers offer multidisciplinary treatment programs with different treatment options, which are adjusted to the patient's needs including physical modalities and exercise, psycho-oncology, nutrition, and information. Early integration of cancer rehabilitation into the cancer care continuum is important because this can significantly improve the quality of patient-centered programs [1-4].

Many cancer survivors are able to benefit from cancer rehabilitation, especially from improvement of physical performance, nutrition, mental stabilization and sufficient pain medicine as well as from longterm prevention and reintegration effects [1-4]. Nevertheless, it is very important to evaluate the patients that have undergone cancer rehabilitation, especially

ao Univ.-Prof. Dr. R. Crevenna, MBA, MSc ( $\triangle)$ Department of Physical Medicine, Rehabilitation and Occupational Medicine, Medical University of Vienna, Waehringer Guertel 18-20, 1090 Vienna, Austria richard.crevenna@meduniwien.ac.at in Austria. This, due to the fact, that (1) cancer rehabilitation has been implemented in Austria only a few years ago and that (2) the existing scientific evidence is week. In their article "Long-term improvement of the bio-psycho-social state of cancer patients after 3 weeks of inpatient oncological rehabilitation: a longterm study at the Humanomed Zentrum Althofen" the authors describe a significant improvement of cancer-associated impairments by a 3-week cancer rehabilitation program, and indicate the impact and socioeconomic importance of cancer rehabilitation. To my opinion, outcome research is a modern, relevant and very important issue for evaluation of cancer rehabilitation, especially concerning sustainability of effects. This outcome research should include longterm analyses of effectiveness and of efficiency of the different parts (exercise, psycho-oncology, nutrition, information) of cancer rehabilitation but also of "3week cancer rehabilitation programs” in total. For the future, applications like online software computerbased health evaluation systems should be used for data collection, administration, and storage of (electronic) patient-reported outcome assessments, and of clinical and sociodemographic data, and furthermore for data import, export, and for graphical presentation of the results in relation to reference values [5]. Due to the fact that many cancer patients are willing and able to work following a cancer diagnosis, and multidisciplinary programs have been shown to help cancer survivors to return to work, evaluation of cancer rehabilitation should also include return-towork outcomes [6]. Furthermore, evaluation should also focus on the effects of rehabilitation programs on bodily, mental and psychosocial functions of elderly (retired and geriatric) cancer patients.

Finally, evaluation of cancer rehabilitation and outcome research in this field should include (objective) clinical data and patient-reported outcome assess- 
ments and focus on effects of rehabilitation on (social and work) reintegration concepts using modern assessment and evaluation systems.

Conflict of interest R. Crevenna declares that he has no competing interests.

\section{References}

1. Mähr B, Keilani M, Wiltschke C, Hassler M, Licht T, Marosi C, Hütterer E, Cenik F, Crevenna R. Cancer rehabilitation in Austria-aspects of physical medicine and rehabilitation. Wien Med Wochenschr. 2016;166(1):39-43. https://doi. org/10.1007/s10354-015-0414-1.

2. Crevenna R. Cancer rehabilitation and palliative care- two important parts of comprehensive cancer care. Support Care Cancer. 2015;23(12):3407-8.https://doi.org/10.1007/ s00520-015-2977-1.
3. Keilani M, Hasenoehrl T, Baumann L, Ristl R, Schwarz M, Marhold M, Sedghi Komandj T, Crevenna R. Effects of resistance exercise in prostate cancer patients: a meta-analysis. Support Care Cancer. 2017;25(9):2953-68. https://doi.org/ 10.1007/s00520-017-3771-z.

4. Keilani M, Hasenoehrl T, Neubauer M, Crevenna R. Resistance exercise and secondarylymphedema in breast cancer survivors-a systematic review. Support Care Cancer. 2016;24(4):1907-16. https://doi.org/10.1007/s00520-0153068-z.

5. Riedl D, Giesinger JM, Wintner LM, Loth FL, Rumpold G, GreilR, NickelsA, LichtT, Holzner B. Improvement of quality of life and psychological distress after inpatient cancer rehabilitation: results of a longitudinal observational study. Wien Klin Wochenschr. 2017;15. https://doi.org/10.1007/ s00508-017-1266-z.

6. Crevenna R. Return-to-work outcomes in cancer survivors. Support Care Cancer. 2017;18. https://doi.org/10.1007/ s00520-017-3835-0. 